\title{
Effectiveness of Chemotherapy for Advanced Adenocarcinoma of the Pancreas in Combined Modality Therapy
}

\author{
Hiroyuki Kato, Hideyuki WaKasugi, Masaki YoKota, Masayuki FuRuKawa, \\ Toshio Mukuta, Yukio Yamada and Akihiro Funakoshi
}

\begin{abstract}
Because it is difficult to diagnose at an early stage, pancreatic carcinoma is usually well advanced by the time it is diagnosed. The combined use of intraoperative radiotherapy, gastrointestinal and/or biliary tract bypass operation, celiac plexus nerve block, and chemotherapy is widely applied in treatment, with favorable results reported in some patients with advanced disease. To evaluate the efficacy of chemotherapy in such combinations, we compared the effect of 5-fluorouracil, its analogues, and mitomycin $C$ on the survival of patients with advanced stage pancreatic cancer. We found no significant difference between the patients treated or not treated with these drugs. Clearly, there is a need for new agents having greater efficacy against pancreatic carcinoma.
\end{abstract}

(Internal Medicine 33: 142-146, 1994)

Key words: 5-fluorouracil, mitomycin C, intraoperative radiotherapy, bypass operation, celiac plexus nerve block

\section{Introduction}

The incidence of adenocarcinoma of the pancreas has steadily risen over the past 40 years $(1,2)$; it is now the fifth leading cause of cancer deaths in Japan. Surgery is the only curative modality for this disease. Even though new imaging methods, e.g., ultrasonography, computed tomography, magnetic resonance imaging, have made diagnosis easier, in many cases, the disease is already well advanced when it is discovered. For unresectable pancreatic cancer, combinations of chemotherapy, radiotherapy, bypass procedure and plexus nerve block are used, with some favorable results reported (3-6). Since there has been no study reported on the efficacy of chemotherapy in such combined therapy, we evaluated the response to 5fluorouracil (5-FU), 5-FU analogues and mitomycin-C (MMC) used in combination modalities in treating patients with advanced pancreatic cancer, by evaluating the survival data.

\section{Patients and Methods}

We studied 49 patients ( 27 men, 22 women, aged 41-80 years) with histologically confirmed carcinoma of the exocrine pancreas. They were selected from 175 consecutive patients with pancreatic carcinoma admitted to the National Kyushu Cancer Center between May 1972 and April 1988. The cases of islet-cell carcinoma were excluded. All 49 had extensive (Stage IV) disease not amenable to surgical resection (7). Gastrointestinal and/or biliary tract bypass operations had been performed in all 49 cases. Other treatments included intraoperative plexus nerve block, intraoperative radiotherapy (IORT) and/or chemotherapy using 5-FU, or a 5-FU analogue (Tegafur, Carmofur, Doxifluridine) with or without MMC as the combined modality therapy. Survival was calculated from the date of operation and was evaluated by the Kaplan-Meier method (8). Statistical differences were calculated by the CoxMantel method $(9,10)$. Differences were considered statistically significant when $\mathrm{p}<0.05$.

\section{Results}

Patients were divided into six groups according to their treatment combination (Table 1). Group 1 consisted of 7 patients who received a bypass operation; Group 2,6 patients who received a bypass operation plus chemotherapy with 5-FU or an analogue; Group 3, 9 patients who received a bypass operation plus chemotherapy with 5-FU or an analogue plus MMC (Table 2 ). The 27 patients treated with both a bypass operation and IORT were divided into three groups: Group 4 (6 patients), Group 5 (12 patients), and Group 6 (9 patients) who received the same combination chemotherapy as Groups 1,2 , and 3 respec-

From the Department of Internal Medicine, National Kyushu Cancer Center, Fukuoka

Received for publication September 22, 1993; Accepted for publication January 22, 1994

Reprint requests should be addressed to Dr. Hiroyuki Kato, the Department of Internal Medicine, Saga Medical School, Nabeshima, Saga 849 


\section{Chemotherapy for Pancreatic Carcinoma}

Table 1. Treatment and Survival of Each Patient Group

\begin{tabular}{ccccccc}
\hline Group & (n) & Bypass procedure & IORT & 5-FU & MMC & $\begin{array}{c}\text { Survival period } \\
\text { (Days) Mean } \pm \text { SD }\end{array}$ \\
\hline 1 & $(7)$ & $(+)$ & & & & $101.8 \pm 26.0$ \\
2 & $(6)$ & $(+)$ & & $(+)$ & & $144.7 \pm 89.4$ \\
3 & $(9)$ & $(+)$ & & $(+)$ & $(+)$ & $102.3 \pm 47.5$ \\
4 & $(6)$ & $(+)$ & $(+)$ & & & $100.7 \pm 59.6$ \\
5 & $(12)$ & $(+)$ & $(+)$ & $(+)$ & & $95.7 \pm 55.3$ \\
6 & $(9)$ & $(+)$ & $(+)$ & $(+)$ & $(+)$ & $149.3 \pm 80.4$ \\
\hline
\end{tabular}

IORT: intraoperative radiotherapy, 5-FU: 5-fluorouracil or its analogue, MMC: mitomycinC.

Table 2. Patients in Groups 1,2 and 3

\begin{tabular}{|c|c|c|c|c|c|c|c|c|}
\hline No. & Age & Sex & Location* & Block** & Surgery & 5-FU & MMC & $\begin{array}{l}\text { Survival Period } \\
\text { (Days) }\end{array}$ \\
\hline \multicolumn{9}{|c|}{ Group 1} \\
\hline 1 & 44 & M & $\mathrm{B}-\mathrm{T}$ & $(-)$ & D-J & & & 91 \\
\hline 2 & 80 & M & B & $(-)$ & CC-J, J-J & & & 112 \\
\hline 3 & 53 & M & $\mathrm{H}$ & $(-)$ & CC-J, J-J & & & 112 \\
\hline 4 & 48 & $\mathrm{~F}$ & B & $(+)$ & G-J & & & 145 \\
\hline 5 & 54 & M & $\mathrm{H}$ & $(-)$ & G-J & & & 94 \\
\hline 6 & 70 & M & B & $(-)$ & G-J & & & 52 \\
\hline 7 & 74 & $\mathrm{M}$ & B & $(+)$ & G-J, CC-J & & & 101 \\
\hline \multicolumn{9}{|c|}{ Group 2} \\
\hline 8 & 60 & M & $\mathrm{H}-\mathrm{B}$ & $(-)$ & CC-J & $\begin{array}{l}5-\mathrm{FU} \\
300 \mathrm{mg} / \text { day }\end{array}$ & & 109 \\
\hline 9 & 65 & $\mathrm{~F}$ & $\mathrm{H}$ & $(+)$ & CC-J & $\begin{array}{l}\text { Carmofur } \\
600 \mathrm{mg} / \text { day }\end{array}$ & & 80 \\
\hline 10 & 56 & $\mathrm{~F}$ & $\mathrm{H}$ & $(+)$ & G-J, CC-J & $\begin{array}{l}5-\mathrm{FU} \\
300 \mathrm{mg} / \text { day }\end{array}$ & & 51 \\
\hline 11 & 70 & $\mathrm{M}$ & $\mathrm{H}$ & $(-)$ & G-J & $\begin{array}{l}\text { Tegafur } \\
800 \mathrm{mg} / \text { day }\end{array}$ & & 327 \\
\hline 12 & 57 & M & $\mathrm{H}$ & $(-)$ & G-J & $\begin{array}{l}\text { Tegafur } \\
800 \mathrm{mg} / \text { day }\end{array}$ & & 136 \\
\hline 13 & 69 & M & $\mathrm{H}$ & $(-)$ & CC-J, J-J & $\begin{array}{l}\text { Tegafur } \\
600 \mathrm{mg} / \text { day }\end{array}$ & & 165 \\
\hline \multicolumn{9}{|c|}{ Group 3} \\
\hline 14 & 63 & M & $\mathrm{H}$ & $(+)$ & G-J, CC-J & $\begin{array}{l}\text { Tegafur } \\
600 \mathrm{mg} / \text { day }\end{array}$ & $20 \mathrm{mg}$ iv & 116 \\
\hline 15 & 72 & $\mathrm{~F}$ & $\mathrm{H}$ & $(-)$ & CC-J & $\begin{array}{l}\text { Tegafur } \\
600 \mathrm{mg} / \text { day }\end{array}$ & $50 \mathrm{mg}$ iv & 122 \\
\hline 16 & 45 & $\mathrm{~F}$ & B & $(-)$ & G-J, CC-J & $\begin{array}{l}\text { Tegafur } \\
600 \mathrm{mg} / \text { day }\end{array}$ & $20 \mathrm{mg}$ iv & 81 \\
\hline 17 & 49 & $\mathrm{~F}$ & B & $(+)$ & G-J, CC-J & $\begin{array}{l}\text { Tegafur } \\
400 \mathrm{mg} / \text { day }\end{array}$ & $20 \mathrm{mg}$ iv & 125 \\
\hline 18 & 65 & M & $\mathrm{H}$ & $(-)$ & CC-J & $\begin{array}{l}\text { Tegafur } \\
600 \mathrm{mg} / \text { day }\end{array}$ & $6 \mathrm{mg}$ iv & 211 \\
\hline 19 & 71 & $\mathrm{~F}$ & $\mathrm{H}$ & $(-)$ & CC-J & $\begin{array}{l}\text { Doxifluridine } \\
600 \mathrm{mg} / \text { day }\end{array}$ & $6 \mathrm{mg}$ iv & 102 \\
\hline 20 & 46 & $\mathrm{~F}$ & $\mathrm{H}-\mathrm{B}$ & $(-)$ & G-J & $\begin{array}{l}5-\mathrm{FU} \\
300 \mathrm{mg} / \text { day }\end{array}$ & $30 \mathrm{mg}$ iv & 56 \\
\hline 21 & 55 & $\mathrm{~F}$ & $\mathrm{H}-\mathrm{B}$ & $(+)$ & G-J, CC-J & $\begin{array}{l}\text { Tegafur } \\
400 \mathrm{mg} / \text { day }\end{array}$ & $10 \mathrm{mg}$ iv & 62 \\
\hline 22 & 57 & F & $\mathrm{H}$ & $(-)$ & G-J, CC-J & $\begin{array}{l}5-\mathrm{FU} \\
250 \mathrm{mg} / \text { day }\end{array}$ & $10 \mathrm{mg}$ iv & 46 \\
\hline
\end{tabular}

*location of the tumor in pancreas (H: head, B: body, T: tail), **celiac plexus nerve block, 5-FU: 5-FU or its analogue, MMC: mitomycin-C, D-J: duodenojejunostomy, CC-J: cholecystojejunostomy, J-J: jejunojejunostomy, G-J: gastrojejunostomy. 


\section{KATO et al}

tively (Table 3). The survival period of each group is shown in paired and the period of survival was examined as shown in Table 1.

Table 4. In pairs I and II, we compared the effect of 5-FU (or its

To evaluate the effect of chemotherapy, the groups were analogue) or of 5-FU (or its analogue) plus MMC in patients

Table 3. Patients in Groups 4, 5 and 6

\begin{tabular}{|c|c|c|c|c|c|c|c|c|c|}
\hline No. & Age & Sex & Location* & Block** & Surgery & $\begin{array}{l}\text { IORT } \\
\text { (Gy) }\end{array}$ & 5-FU & MMC & $\begin{array}{l}\text { Survival period } \\
\text { (Days) }\end{array}$ \\
\hline \multicolumn{10}{|c|}{ Group 4} \\
\hline 23 & 50 & $\mathrm{M}$ & $\mathrm{H}$ & $(-)$ & CC-J & 30 & & & 203 \\
\hline 24 & 49 & $\mathrm{~F}$ & $\mathrm{H}$ & $(+)$ & G-J, CD-J & 20 & & & 147 \\
\hline 25 & 62 & $\mathrm{M}$ & $\mathrm{H}$ & $(+)$ & CD-J & 20 & & & 43 \\
\hline 26 & 61 & $\mathrm{~F}$ & $\mathrm{H}$ & $(-)$ & G-J & 20 & & & 38 \\
\hline 27 & 73 & F & $\mathrm{T}$ & $(+)$ & CC-J & 30 & & & 109 \\
\hline 28 & 58 & F & $\mathrm{H}$ & $(+)$ & CD-J, J-J & 25 & & & 64 \\
\hline \multicolumn{10}{|c|}{ Group 5} \\
\hline 29 & 62 & M & $\mathrm{H}-\mathrm{B}$ & $(+)$ & G-J & 27 & $\begin{array}{l}\text { Doxifluridine } \\
1,200 \mathrm{mg} / \text { day }\end{array}$ & & 59 \\
\hline 30 & 71 & M & $\mathrm{B}-\mathrm{T}$ & $(+)$ & G-J & 40 & $\begin{array}{l}\text { Tegafur } \\
600 \mathrm{mg} / \text { day }\end{array}$ & & 199 \\
\hline 31 & 72 & M & B & $(+)$ & G-J, CC-J & 40 & $\begin{array}{l}\text { Tegafur } \\
600 \mathrm{mg} / \text { day }\end{array}$ & & 110 \\
\hline 32 & 66 & $\mathrm{~F}$ & B & $(-)$ & G-J & 35 & $\begin{array}{l}\text { Tegafur } \\
600 \mathrm{mg} / \text { day }\end{array}$ & & 137 \\
\hline 33 & 61 & M & B & $(-)$ & CD-J, CC-J & 20 & $\begin{array}{l}5-\mathrm{FU} \\
250 \mathrm{mg} / \text { day }\end{array}$ & & 126 \\
\hline 34 & 80 & M & $\mathrm{H}$ & $(-)$ & G-J & 30 & $\begin{array}{l}\text { Tegafur } \\
600 \mathrm{mg} / \text { day }\end{array}$ & & 92 \\
\hline 35 & 55 & $\mathrm{~F}$ & $\mathrm{H}$ & $(-)$ & G-J & 18 & $\begin{array}{l}\text { Tegafur } \\
400 \mathrm{mg} / \text { day }\end{array}$ & & 69 \\
\hline 36 & 69 & F & $\mathrm{H}$ & $(-)$ & G-J, CC-J & 30 & $\begin{array}{l}\text { Carmofur } \\
200 \mathrm{mg} / \text { day }\end{array}$ & & 32 \\
\hline 37 & 69 & M & B & $(-)$ & G-J, CC-J & 20 & $\begin{array}{l}\text { Tegafur } \\
600 \mathrm{mg} / \text { day }\end{array}$ & & 189 \\
\hline 38 & 47 & $\mathrm{~F}$ & B & $(+)$ & G-J, CC-J & 15 & $\begin{array}{l}\text { Tegafur } \\
800 \mathrm{mg} / \text { day }\end{array}$ & & 67 \\
\hline 39 & 41 & M & B & $(-)$ & CD-J & 25 & $\begin{array}{l}\text { Tegafur } \\
600 \mathrm{mg} / \text { day }\end{array}$ & & 34 \\
\hline 40 & 48 & M & $\mathrm{H}-\mathrm{B}$ & $(+)$ & G-J & 20 & $\begin{array}{l}\text { Carmofur } \\
600 \mathrm{mg} / \text { day }\end{array}$ & & 34 \\
\hline \multicolumn{10}{|c|}{ Group 6} \\
\hline 41 & 72 & M & B & $(-)$ & CC-J & 25 & $\begin{array}{l}\text { Tegafur } \\
1,200 \mathrm{mg} / \text { day }\end{array}$ & $10 \mathrm{mg}$ iv & 235 \\
\hline 42 & 63 & $\mathrm{M}$ & $\mathrm{B}-\mathrm{T}$ & $(+)$ & G-J & 30 & $\begin{array}{l}5-\mathrm{FU} \\
200 \mathrm{mg} / \text { day }\end{array}$ & $10 \mathrm{mg}$ iv & 90 \\
\hline 43 & 69 & M & B & $(+)$ & G-J, CC-J & 20 & $\begin{array}{l}\text { Tegafur } \\
600 \mathrm{mg} / \text { day }\end{array}$ & $10 \mathrm{mg}$ iv & 179 \\
\hline 44 & 57 & $\mathrm{~F}$ & $B-T$ & $(+)$ & CC-J & 30 & $\begin{array}{l}\text { Tegafur } \\
400 \mathrm{mg} / \text { day }\end{array}$ & $10 \mathrm{mg}$ iv & 83 \\
\hline 45 & 75 & $\mathrm{~F}$ & $\mathrm{~B}-\mathrm{T}$ & $(+)$ & CC-J & 20 & $\begin{array}{l}\text { Tegafur } \\
400 \mathrm{mg} / \text { day }\end{array}$ & $8 \mathrm{mg}$ iv & 79 \\
\hline 46 & 68 & M & $\mathrm{H}$ & $(+)$ & G-J, CC-J & 25 & $\begin{array}{l}\text { Carmofur } \\
300 \mathrm{mg} / \text { day }\end{array}$ & $10 \mathrm{mg}$ iv & 105 \\
\hline 47 & 62 & M & B & $(+)$ & CC-J & 35 & $\begin{array}{l}\text { Tegafur } \\
600 \mathrm{mg} / \text { day }\end{array}$ & $20 \mathrm{mg}$ iv & 116 \\
\hline 48 & 72 & $\mathrm{~F}$ & $\mathrm{H}$ & $(-)$ & G-J & 30 & $\begin{array}{l}5-\mathrm{FU} \\
300 \mathrm{mg} / \text { day }\end{array}$ & $8 \mathrm{mg}$ iv & 332 \\
\hline 49 & 63 & $\mathrm{~F}$ & B & $(+)$ & G-J, CC-J & 25 & $\begin{array}{l}\text { Tegafur } \\
600 \mathrm{mg} / \text { day }\end{array}$ & $10 \mathrm{mg}$ iv & 125 \\
\hline
\end{tabular}

*location of the tumor in pancreas (H: head, B: body, T: tail), **celiac plexus nerve block, IORT: intraoperative radiotherapy, 5-FU: 5-FU or its analogue, MMC: mitomycin-C, CC-J: cholecystojejunostomy, G-J: gastrojejunostomy, CD-J: choledochojejunostomy, J-J: jejunojejunostomy. 


\section{Chemotherapy for Pancreatic Carcinoma}

who received the bypass operation. In pairs III and IV, we compared the effect of 5-FU (or its analogue) or 5-FU (or its analogue) plus MMC in patients who received a bypass operation plus IORT. The Kaplan-Meier survival plots for these pairs are shown in Fig. 1 and 2. No significant difference in survival was found in any of the pairs (I-IV).

Table 4. Group Pairs Used in Comparing Survival

\begin{tabular}{cccc}
\hline Pair No. & Group & & Group \\
\hline I & 1 & vs & 2 \\
II & 1 & vs & 3 \\
III & 4 & vs & 5 \\
IV & 4 & vs & 6 \\
\hline
\end{tabular}

vs: versus.

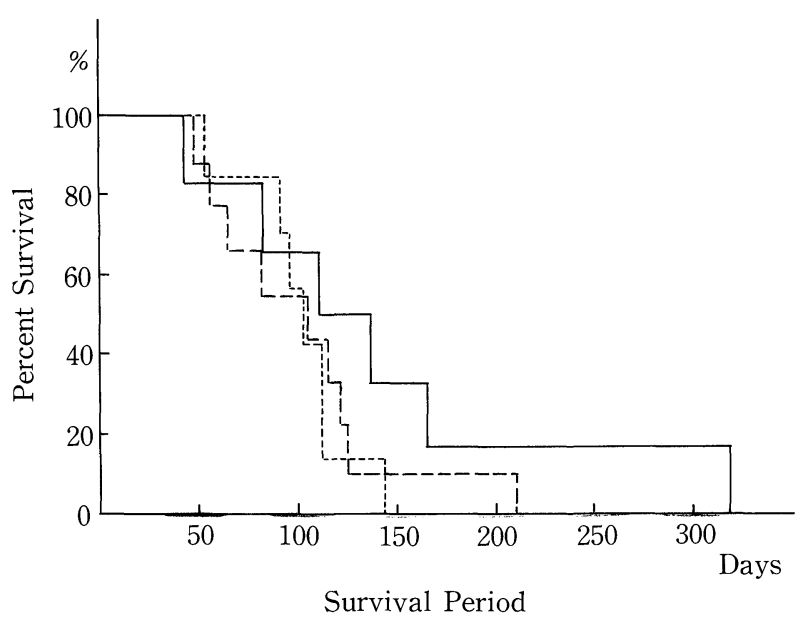

Fig. 1. Survival curves for Group 1, Group 2 and Group 3 patients. ------ Group 1, — Group 2, ----Group 3.

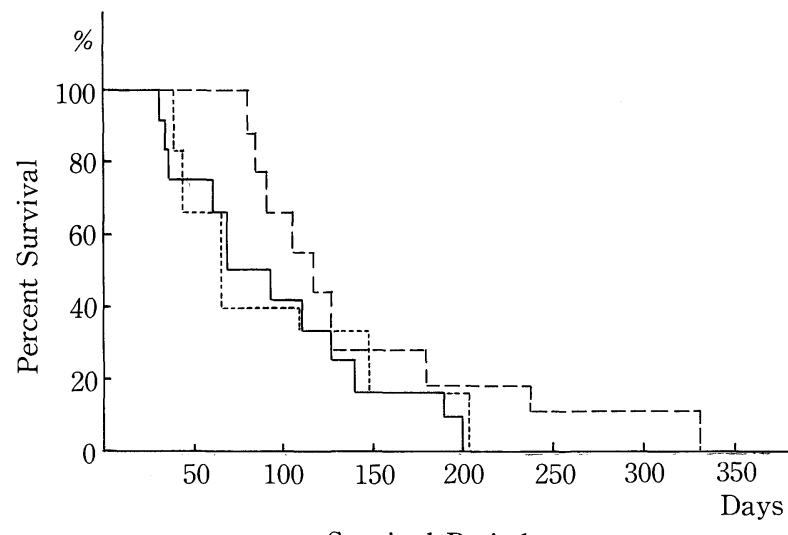

Survival Period

Fig. 2. Survival curves for Group 4, Group 5 and Group 6 patients. Group 4, - Group 5, ---- Group 6.

\section{Discussion}

Even though imaging techniques such as ultrasonography, computed tomography and magnetic resonance have made the diagnosis of pancreatic cancer easier, the disease is usually advanced by the time it is discovered. Involvement of major abdominal vasculature or lymph nodes makes many patients unsuitable candidates for definite surgery. In such patients with advanced disease, combined modality therapy may include IORT, a gastrointestinal and/or biliary tract bypass operation, celiac plexus nerve block and chemotherapy (3-6). Since Abe and Takahashi (3) reported the effectiveness of IORT, it has been performed at our hospital. With this method, a 15-40 Gy "boost" is delivered directly to the surgically exposed tumor by an electron beam through a field-limiting cone, thus avoiding injury to neighboring tissue. Dobelbower and colleagues reported data from 720 cases with unresectable pancreatic cancer treated with IORT. Median patient survival time was 5.8-13.5 months (11).

5 -FU is the most extensively studied chemotherapeutic agent in pancreatic cancer. In Japan, 5-FU and its analogues, Tegafur, Carmofur, and Doxifluridine, are administered orally as maintenance therapy after surgery in the hope of prolonging survival. However, an increase in survival has not been confirmed when chemotherapy has been used in combined modality therapy. In the present study, we statistically evaluated the effect of 5-FU, its analogues, and MMC using survival curves, and found no significant differences in survival between patients who were taking these drugs and those who were not. The advanced islet-cell carcinoma was excluded from the present study, because this tumor frequently shows indolent progression even after metastasis has occurred (12).

Many agents have been used to combat pancreatic cancer, including 5-FU, MMC, adriamycin and streptozocin. Carter and Comis (13), analyzing 212 patients treated with 5-FU, reported an overall positive response rate of $28 \%$. The antibiotic, MMC, given as monotherapy is to exhibit activity comparable to that of 5-FU; 12 of 44 patients $(27 \%)$ achieved a response (14). The introduction of new chemotherapeutic agents has progressed slowly, in part because the response rate is highly variable. For instance, in trials with $5-\mathrm{FU}$, responses have ranged from $0 \%$ to $67 \%$. This wide range has variously been attributed to differences in patient selection, criteria used to define a response, or the small number of patients studied in uncontrolled trials. However, the most recent review indicates that 5-FU is the only agent with a response rate exceeding $20 \%$ (15). This response rate is not very high. Moreover, no standard chemotherapy regimen for pancreatic cancer has been established.

We conclude that chemotherapy using 5-FU, or its analogues, and MMC failed to prolong the survival of patients with advanced adenocarcinoma of the pancreas. We urge efforts be renewed to develop more effective agents for use as combined modality therapy for patients with pancreatic cancer. 


\section{KATO et al}

Acknowledgements: This study was supported in part by a cancer research grant from the Ministry of Health and Welfare, Japan.

\section{References}

1) Ishii K. Pancreatic cancer. Jpn J Clin Med 32: 2267, 1974 (in Japanese).

2) Hirayama $T$. The epidemiologic study of pancreatic cancer. Kan Tan Sui (Japan) 4: 477, 1982 (in Japanese).

3) Abe M, Takahashi M. Intraoperative radiotherapy: the Japanese experience. Int J Radiation Oncology Biol Phys 7: 863, 1981.

4) Yasue M, Morimoto T, Ochiai E, et al. Combined modality therapy for stage IV advanced pancreatic cancer with special reference to intraoperative radiotherapy (IOR). Jpn J Gastroenterol Surg 16: 1866, 1983 (in Japanese).

5) Goldson AL, Ashaveri E, Espinoza MC, et al. Single high dose intraoperative electrons for advanced stage pancreatic cancer: phase I pilot study. Int J Radiation Oncology Biol Phys 7: 869, 1981.

6) Wood WC, Shipley WU, Gunderson LL, Cohen AM, Nardi GL. Intraoperative irradiation for unresectable pancreatic carcinoma. Cancer 49: $1272,1982$.

7) Japanese Pancreatic Society. General rules for surgical and pathological studies on cancer of pancreas, 2nd Ed. Kanehara Ltd., Tokyo, 1982.

8) Kaplan EL, Meier P. Nonparametric estimation from incomplete observations. J Am Stat Assoc 53: 457, 1958.

9) Cox DR. Regression models and life-tables. J R Statist Soc B 34: 187, 1972.

10) Mantel N. Evaluation of survival data and two new rank order statistics arising in its consideration. Cancer Chemother Rep 50: 163, 1966.

11) Dobelbower RR Jr, Konski AA, Merrick HW III, Bronn DG, Schifeling $\mathrm{D}$, Kamen C. Intraoperative electron beam radiation therapy (IOEBRT) for carcinoma of the exocrine pancreas. Int J Radiation Oncology Biol Phys 20: 113, 1991.

12) MoertelCG,Lefkopoulo M,LipsitzS,Hahn RG, Klaassen D. Streptozocindoxorubicin, streptozocin-fluorouracil, or chlorozotocin in the treatment of advanced islet-cell carcinoma. N Engl J Med 326: 519, 1992.

13) Carter SK, Comis RL. The integration of chemotherapy into a combined modality approach for cancer treatment. VI. Pancreatic adenocarcinoma. Cancer Treat Rev 2: 193, 1975.

14) Harvey JH, Schein PS. Chemotherapy of pancreatic carcinoma. World J Surg 8: 935, 1984.

15) Warshaw AL, Castillo CF. Pancreatic carcinoma. N Engl J Med 326: 455, 1992. 